\title{
Benthic assemblages in two Mediterranean caves: species diversity and coverage as a function of abiotic parameters and geographic distance
}

\author{
Ruth Martí* ${ }^{\ddagger}$, Maria J. Uriz*, Enric Ballesteros* and Xavier Turon ${ }^{\dagger}$ \\ *Centre d'Estudis Avançats de Blanes, CSIC, Accés Cala Sant Francesc, 14, 17300 Blanes (Girona), Spain. \\ ${ }^{\dagger}$ Department of Animal Biology, Faculty of Biology, University of Barcelona, Diagonal Ave. 645, 08028 Barcelona, Spain. \\ ${ }^{\ddagger}$ Corresponding author, e-mail: ruth@ceab.csic.es
}

\begin{abstract}
Benthic assemblages of two Mediterranean submarine caves were compared. Species coverage and number of species were lower in internal (dark) communities than external. This feature was specially marked in the less illuminated cave. Ordination analyses performed on species coverage per community for each cave separately, distinguished several benthic communities from the outermost to the innermost zone of each cave. Cluster analyses on species coverage, taking into account all communities in both the caves, established similarities among communities: algal-dominated communities clustered according to the level of light received independently of the cave they inhabited, while animal-dominated communities were more similar within each cave than between the caves. Moreover, among the abiotic parameters measured irradiance was the only factor that clearly diminished from the entrance to the innermost zone in both the caves. In contrast, water movement and particulate organic matter varied differently in each cave. Results indicate that the different topography, depth and geographic location of the two caves determine water movement, light penetration and nutrient availability along the caves. These factors are responsible for determining species abundance and diversity, as well as species growth habit in each community.
\end{abstract}

\section{INTRODUCTION}

Submarine caves show patterns comparable worldwide in structure and type of communities. A common feature is a decrease in species richness, biomass and coverage of benthic organisms from the outermost to the innermost part of the cave (see Harmelin, 1985 and references therein). The usual explanation for this gradient is a reduced water turnover towards the inner part (Fichez, 1991), although experiments comparing the dissolution of plaster balls failed to substantiate this hypothesis (Balduzzi et al., 1989; Zabala et al., 1989). Likewise, no stagnation of water in microlayers over wall boundaries has been detected by dye diffusion (Zabala et al., 1989). Other physico-chemical gradients (salinity, temperature, density, dissolved oxygen and chlorophyll) that could explain this zonation were not detected either (Gili et al., 1986; Zabala et al., 1989). Light is, in all cases, the factor that clearly decreases from the external to the innermost zone of all the caves. For planktonic organisms, a strong decrease in the number of individuals from external to internal zones reported in a Mediterranean cave (Palau et al., 1991) may be explained by simple diffusionsedimentation processes (Garrabou \& Flos, 1995). However, this result is inconsistent with the lack of decrease in particulate organic matter (POM) reported for the same cave (Gili et al., 1986; Zabala et al., 1989), although it might be explained by the importance of the tripton part of the seston (not considered in planktonic studies) (Palau et al., 1991). Moreover, it has been suggested that the organic content of the particles inside the caves is lower than that of the particles outside them, so the quality of the food supply potentially available to suspension-feeders would be higher in the external zones of the caves (Fichez, 1991).

Here we attempt to compare species coverage and richness of the flora and fauna in the different communities established in two Mediterranean caves. Using photography we were able to study large areas in the caves and to encompass spatial heterogeneity. Moreover, values of abiotic parameters (irradiance, water movement and POM) were obtained for comparative purposes. Samples were collected in spring and autumn to allow for possible seasonal variation in species presence and/or abundance.

From the comparison of these two caves with contrasting topographical and trophic conditions we aimed to identify the factors determining community structure and variation along the caves, and establish whether the seasonality reported in open littoral communities of the Mediterranean also applies to the inner communities of the caves.

\section{MATERIALS AND METHODS}

Study sites

The caves are located in the north-western Mediterranean (Iberian Peninsula): the Cabrera Archipelago (Balearic Islands, oligotrophic sea) and the Medes Islands (Catalan coast, a relatively eutrophic zone). The two caves (Figure 1) have in common the limestone nature of the substrate and the mainly rocky nature 


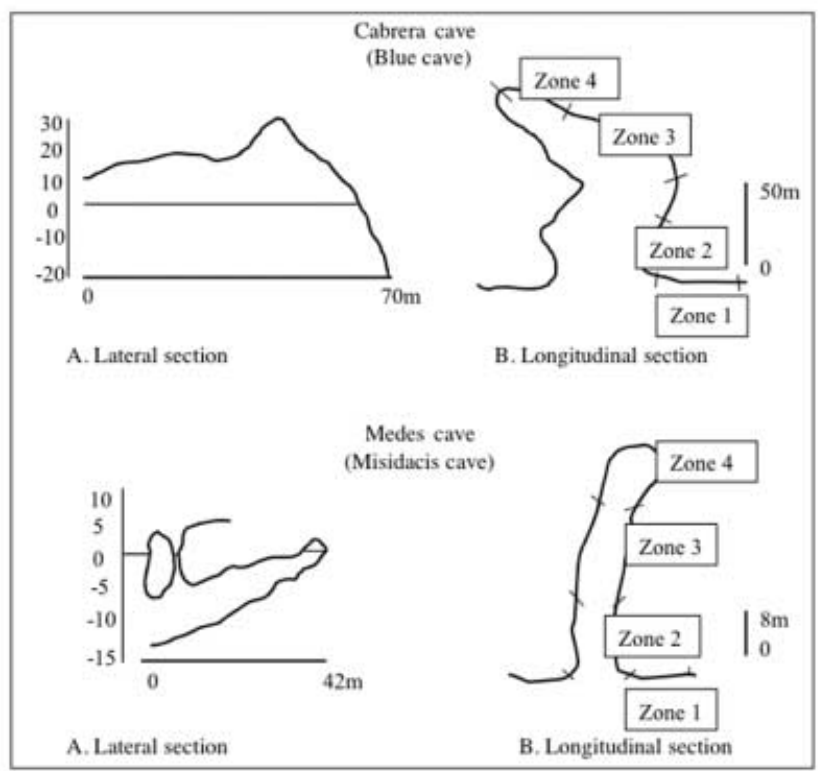

Figure 1. Cave morphology and location of the zones studied.

of the floor, with low amounts of sediment, in contrast to what is commonly found in the caves. They differ in topography and trophic characteristics.

\section{Assemblage assessment}

A longitudinal, $2 \mathrm{~m}$ wide transect was set up from the cave entrance to the innermost zone in both the caves. The species inventories and the measurement of physical parameters were performed by SCUBA diving along each transect. In the Cabrera cave the transect was located at $6 \mathrm{~m}$ depth and in the Medes cave at about $\sim 10 \mathrm{~m}$ depth, which represented the mean depth of the respective caves. The study was performed in June and November 1996.

A semi-quantitative inventory of the benthic species was drawn up according to the method described in Braun Blanquet (1979). Species abundance was assessed in situ, and samples were collected when necessary for later taxonomic identification in the laboratory.

The semi-quantitative inventories distinguished several communities throughout the caves. Once the main communities (zones) had been established, the physical and biological parameters of each zone were studied.

\section{Physical parameters \\ Irradiance}

Light in each zone was measured in June and November using a SPQA Li-Cor provided with a data-logger Li-Cor LI-1000. The values are referred to the subsurface light.

\section{Water motion and temperature}

Water motion in the established zones of both the caves was derived from measurement of weight losses of calcium sulphate balls $(1.5 \mathrm{~cm}$ in diameter) (Muus, 1968). A wire was passed through each ball and the wires were included in a flexible resin, which was attached to the walls. In a variety of sea conditions, four sets of five balls were maintained for two days on the walls of each zone, in June and November. Five additional balls were left underwater in the cave in a closed container for the same period, as a control for $\mathrm{CaSO}_{4}$ dissolution in static conditions. Balls were dried for $24 \mathrm{~h}$ at $100^{\circ} \mathrm{C}$ after collection and then weighed. Losses of $\mathrm{CaSO}_{4}$ in control balls were subtracted from losses measured in the balls placed on the walls. The results are expressed in $\mathrm{mg}$ of $\mathrm{CaSO}_{4}$ lost per hour.

Water temperature was measured in each zone in both seasons.

\section{Particulate organic matter (POM)}

Seawater was collected with a Niskins sampler in each zone of both the caves, in June and November. Two litres of water were filtered on precombusted $\mathrm{GF} / \mathrm{F}$ glass fibre filters of $0.22-\mu \mathrm{m}$ pore diameter. Afterwards, filters were exposed to hydrochloric acid vapour for $48 \mathrm{~h}$ to eliminate the inorganic material, dried and analysed with a $\mathrm{C}: \mathrm{H}: \mathrm{N}$ Autoanalyser Eager 200. Final concentrations are expressed in $\mu \mathrm{g} /$ litre.

\section{Community structure Sampling}

To assess seasonal coverage of the most abundant species, 20 pictures were taken at random in each zone in both the caves, in June and November 1996. The pictures, covering $\sim 310 \mathrm{~cm}^{2}$ each, were taken with a Nikonos-V camera and a $28 \mathrm{~mm}$ objective provided with a Nikonos close-up lens.

All seaweeds and animals in each picture were outlined and identified to the species level (whenever possible). The outlines were later digitalized and analysed with Sigma Scan Image software (Jandel) to measure the total area and number of individuals of each species (which corresponds to the number of patches for sponges and other encrusting organisms).

\section{Coverage and number of species}

The percentage cover of each species in the 20 pictures taken per zone and season was calculated. The values were added to obtain the percentage cover of each species in the whole area sampled per zone $\left(6200 \mathrm{~cm}^{2}\right)$. A log-linear model comparing the number of species per taxon in each zone was performed for each cave.

\section{Ordination}

Detrended correspondence analyses (DCAs) were performed on species coverage to study the spatial distribution of the benthic species in each of the four zones previously defined in both the caves. This analysis provided a graph of the distance(s) between the zones on the basis of differences in species composition and abundance. The percentage cover per species was the variable analysed. The percentage of bare rock was also taken into account, since it varied strongly between zones.

The analyses used the software program PC-ORD version 4 (McCune \& Mefford, 1999). Outliers were 
previously eliminated using the Sorensen (Bray-Curtis) distance. The down weighting of rare species option was selected to avoid their possible influence on ordination.

Because species coverage varied from June to November, especially in the most external zones dominated by seaweeds, the ordination analyses were carried out separately for each sampling season. However, as the general pattern was similar in both seasons, only the analyses from June are shown here.

An analysis was first performed for all samples of each cave separately (80 samples, 20 pictures per zone). A second analysis was performed for the animal-dominated zones (Zones 3 and 4 of the Cabrera cave and Zones 2, 3 and 4 of the Medes cave) of each cave separately since they appeared clumped in the first analysis.

\section{Classification}

Cluster analyses were conducted on percentage cover per taxonomic group to assess the relationships between zones of the two caves in the two seasons. Bare rock was not considered for these analyses.

Hierarchical clustering with group-average linking, based on similarity matrices (Bray-Curtis coefficient) was used. Data were previously transformed to double root (4th root), which down weights the importance of the very abundant species so that the less dominant, and even the rare species, contribute to similarity between samples. The PRIMER (Plymouth Routines in Multivariate Ecological Research) package for the analyses (Clarke \& Warwick, 1994) was used.

A preliminary analysis was done using the percentage cover per high taxonomic entity (from Phylum to Class depending on the group) as the variable. Using the groups found in this analysis, more accurate analyses were carried out on the percentage cover per species.

\section{RESULTS}

Assemblage assessment

Species composition and abundance (semi-quantitative inventories) as well as changes in growth habit of the same species (for example massive forms at the cave entrance becoming thinly encrusting forms at the innermost parts), allowed us to distinguish four differentiated zones in each cave, which corresponded to four different communities (Pérès \& Picard, 1964).

In the Cabrera cave (Figure 1A), Zone 1 corresponded to a photophilic seaweed community, and Zone 2 to a sciaphilic seaweed community. The former, facing west, was located just outside the cave. The sciaphilic community faced north-west and was located at the cave entrance. Zone 3 corresponded to a semi-dark cave community and Zone 4 represented a dark community in several aspects (see Discussion) (terminology according to Pérès \& Picard, 1964).

The narrow shape of the Medes (Figure 1B) cave and its deeper location as compared to the Cabrera cave results in a darker nature of its communities. Zone 1 faced south-east out of the cave and had a hemisciaphilic seaweed community. The communities inhabiting Zones 2 and 3 corresponded to a semi-dark cave community. Although Zone
3 was darker, both zones had similar species composition, although the species showed different growth habits. The assemblage dwelling on Zone 4 represented a dark-cave community. The cave ended with a zone almost free of macroinvertebrates.

The three communities of seaweeds in the two caves can be therefore positioned along a gradient of light: photophilic, hemisciaphilic and sciaphilic.

\section{Physical parameters \\ Irradiance}

The irradiance values in each zone of both the caves are reported in Table 1. In the Cabrera cave, Zone 1 (located outside the cave) was well illuminated (Table 1). Zone 2 received nearly $3 \%$ of the subsurface light. In the two innermost zones no light was detected at 11 a.m. At sunset some light was detected in Zone 4 due to the west facing of the cave.

The two innermost zones of the Medes cave were dark throughout the day (Table 1). Zone 2 received some light from a pit that opens to the outside just above it and from the entrance to the cave. Zone 1, although it was placed outside the cave (as in the Cabrera cave), received less light than Zone 1 in the Cabrera cave, since it was deeper.

\section{Water motion and temperature}

In the Cabrera cave, Zone 3 had the lowest water motion in both seasons while Zone 2 showed the highest

Table 1. Irradiance in each zone of both the caves. The mean values are referred to the subsurface light $(0 \mathrm{~m}$ depth). There were no appreciable differences between seasons.

\begin{tabular}{lccc}
\hline \multirow{2}{*}{ Season } & & \multicolumn{2}{c}{$\%$ of irradiance } \\
\cline { 3 - 4 } & Zone & Cabrera cave & Medes cave \\
\hline June & 1 & 42.8 & 19.5 \\
and & 2 & 2.7 & 0.076 \\
November & 3 & 0.0036 & 0 \\
& 4 & 0 & 0 \\
\hline
\end{tabular}

Table 2. Water motion expressed in $\mathrm{mg} \mathrm{CaSO}_{4}$ lost per hour. The values correspond to means and standard errors of four sets of measures per season including different sea conditions.

\begin{tabular}{|c|c|c|}
\hline \multirow[b]{2}{*}{ Season and zone } & \multicolumn{2}{|c|}{$\mathrm{mg} \mathrm{CaSO} / \mathrm{h}$} \\
\hline & Cabrera cave & Medes cave \\
\hline \multicolumn{3}{|l|}{ June } \\
\hline 1 & $125.6( \pm 38.6)$ & $293.5( \pm 8.9)$ \\
\hline 2 & $175.2( \pm 75.5)$ & $101.0( \pm 17.1)$ \\
\hline 3 & $90.2( \pm 31.4)$ & $116.0( \pm 20.1)$ \\
\hline 4 & $117.5( \pm 56.1)$ & $160.3( \pm 27.3)$ \\
\hline \multicolumn{3}{|l|}{ November } \\
\hline 1 & $129.2( \pm 46.3)$ & $145.4( \pm 49.5)$ \\
\hline 2 & $184.9( \pm 75.1)$ & $35.0( \pm 21.2)$ \\
\hline 3 & $70.0( \pm 31.5)$ & $40.3( \pm 27.0)$ \\
\hline 4 & $151.3( \pm 45.5)$ & $50.6( \pm 31.8)$ \\
\hline
\end{tabular}


Table 3. Particulate organic carbon and nitrogen $(\mu \mathrm{g} / \mathrm{l})$ in the water in the four zones of the two caves and in the two seasons.

\begin{tabular}{|c|c|c|c|c|}
\hline \multirow[b]{2}{*}{ Season and zone } & \multicolumn{2}{|c|}{ Cabrera cave } & \multicolumn{2}{|c|}{ Medes cave } \\
\hline & Carbon & Nitrogen & Carbon & Nitrogen \\
\hline \multicolumn{5}{|l|}{ June } \\
\hline 1 & 834 & 76 & 1175 & 67 \\
\hline 2 & 818 & 88 & 1206 & 151 \\
\hline 3 & 1226 & 87 & 1614 & 104 \\
\hline 4 & 1251 & 81 & 1313 & 113 \\
\hline \multicolumn{5}{|l|}{ November } \\
\hline 1 & 896 & 93 & 2102 & 210 \\
\hline 2 & 870 & 89 & 2250 & 256 \\
\hline 3 & 1282 & 101 & 2495 & 317 \\
\hline 4 & 1336 & 99 & 2284 & 286 \\
\hline
\end{tabular}

(Table 2). Zones 1 and 4 were always within the range of values of Zones 2 and 3. However, while water movement in Zone 1 was comparable in both seasons, water motion in Zone 4 was higher $(\sim 30 \%)$ in November than in June.

In the Medes cave, comparison between seasons was precluded due to very different sea conditions (much rougher in June). The zone outside the cave (Zone 1) always had the highest water motion, followed by the innermost zone. Zones 3 and 2 had similar water motion, in both cases lower than Zones 1 and 4 .

The temperature values registered indicated that there were no differences between zones or seasons within each cave. The average temperature was $19^{\circ} \mathrm{C}$ in the Cabrera cave and $17^{\circ} \mathrm{C}$ in the Medes cave.

\section{Particulate organic matter (POM)}

The results shown in Table 3 for POM content are merely illustrative, since water samples were taken only once per season. The values, however, are useful for assessing within-cave trends.

In the Cabrera cave, the organic matter concentration was similar in both seasons for all zones. Nitrogen concentration, as expected, was lower in absolute values than carbon concentration. Carbon concentration was higher in the two innermost zones of the cave than in the two external ones.

In the Medes cave, a gradient of POM along the cave was evident. Zone 3 had the highest concentration of carbon, followed by Zone 4, Zone 2 and finally Zone 1. Nitrogen concentration was also lower outside the cave (Zone 1), while inside the cave Zone 2 showed the highest value. Concentrations were higher in November than in June.

\section{Community structure Coverage}

The coverage of all species from the two caves is listed in Table 4 (see Appendix for list of species and authorities).

In the Cabrera cave there were no outstanding differences in species coverage between seasons in any zone (Figure 2). Zone 1 was clearly dominated by seaweeds and the substratum was totally covered. In Zone 2, although seaweeds were still the dominant group and the substratum was also totally covered, other taxonomic groups (sponges, cnidarians and bryozoans) represented $12-18 \%$ of the total coverage. Zones 3 and 4 lacked seaweeds and were dominated by filter- and suspensionfeeders. In both zones a large amount of bare rock was present, being more abundant in Zone 4.

The differences between Zones 3 and 4 were mainly due to the faunal composition. While in Zone 3, bryozoans, cnidarians and sponges were abundant, the last group was the most important in terms of coverage. In Zone 4 sponges clearly dominated and cnidarians and bryozoans were poorly represented.

In the Medes cave (Figure 2) there were large differences in taxon abundance with respect to the Cabrera cave. Only the external zone (1) was dominated by seaweeds. The remaining zones inside the cave were dominated by animals. The traits common to the internal Zones $(2,3$, and 4$)$ were the dominance of sponges and the high proportion of bare rock (up to $73 \%$ in June in Zone 4), although their respective percentages varied among zones.

When comparing the caves, differences became evident. First, Zones 1 and 2 in the Cabrera cave were dominated by seaweeds while only Zone 1 was in the Medes cave.

Zones 2, 3, and 4 of the Cabrera cave were roughly equivalent in composition to Zones 1, 2, and 3 of the Medes cave, respectively. Finally, the equivalent of Zone 4 from the Medes cave was not present in the Cabrera cave. The low animal cover of Zone 4 in the Medes cave was an indicator of its extreme confinement.

\section{Number of species}

In the Cabrera cave (Figure 3) the differences in the number of species between the two external zones (1 and 2 ) and the similarity in this parameter in the two internal zones ( 3 and 4) are noteworthy. This pattern was not observed for the percentage cover (Figure 2). A log-linear model comparing the number of species per taxon in each zone detected significant $(P<0.001)$ differences in species composition between zones. In Zone 1 the number of seaweed species was higher in both seasons than that of the remaining taxonomic groups. Moreover, in November there were ten more seaweed species than in June. In Zone 2 seaweeds were also the dominant group in number of species but the sum of animal species was higher than in Zone 1. In November the number of seaweed species decreased with respect to June. Zones 3 and 4 were similar with the dominant groups, ranked from the richest to the poorest, being: sponges, bryozoans, cnidarians and polychaetes. The number of sponge species in Zone 4 was the highest but no noticeable differences were found for other taxonomic groups.

In the Medes cave (Figure 3), the similarity of the three internal zones (2, 3 and 4), which clearly differ from the external zone (1), is evident. As in the Cabrera cave, a log-linear model comparing the number of species per taxon in each zone revealed a significant $(P<0.001)$ difference between zones. In Zone 1 , the number of seaweed species equalled the sum of the species of the other taxonomic groups; in both seasons algae were the dominant group followed by sponges. In Zones 2, 3 and 4, sponges were the group with the highest number of species and Zone 2 had the highest value. Bryozoans, cnidarians and 
Table 4. Species coverage (\%) from 20 pictures taken at random per zone in the Cabrera cave and in the Medes cave in $\mathrm{Fune}$ and November.

\begin{tabular}{|c|c|c|c|c|c|c|c|c|}
\hline \multirow{2}{*}{$\begin{array}{l}\text { Species } \\
\text { Cabrera cave }\end{array}$} & \multicolumn{4}{|c|}{ June } & \multicolumn{4}{|c|}{ November } \\
\hline & Zone 1 & Zone 2 & Zone 3 & Zone 4 & Zone 1 & Zone 2 & Zone 3 & Zone 4 \\
\hline \multicolumn{9}{|l|}{ Chlorophyta } \\
\hline Acetabularia acetabulum & 0.01 & & & & & & & \\
\hline Acetabularia parvula & & & & & 0.01 & & & \\
\hline Anadyomene stellata & 0.15 & & & & 0.35 & & & \\
\hline Cladophora pellucida & & 0.09 & & & & & & \\
\hline Cladophora sp. & & & & & 0.15 & 0.08 & & \\
\hline Codium bursa & 0.04 & & & & 0.25 & & & \\
\hline Flabellia petiolata & 0.09 & 5.39 & & & 0.54 & 8.34 & & \\
\hline Halimeda tuna & 0.03 & 0.02 & & & 0.05 & & & \\
\hline Palmophyllum crassum & & 6.16 & & & & 5.78 & & \\
\hline Pseudochlorodesmis furcellata & 0.12 & 1.09 & & & 0.01 & & & \\
\hline Valonia utricularis & & 0.06 & & & 0.01 & 0.03 & & \\
\hline \multicolumn{9}{|l|}{ Phaeophyta } \\
\hline Aglaozonia sp. & & 0.02 & & & & & & \\
\hline Cystoseira balearica & 10.63 & & & & 2.06 & & & \\
\hline Cystoseira compressa & 0.65 & & & & 0.01 & & & \\
\hline Dictyopteris polypodioides & 2.73 & & & & 2.94 & 0.002 & & \\
\hline Dictyota dichotoma & 0.20 & 0.03 & & & 0.14 & & & \\
\hline Dictyota dichotoma var. intricata & 23.81 & & & & 2.51 & & & \\
\hline Halopteris filicina & & 3.74 & & & & 1.03 & & \\
\hline Halopteris scoparia & 15.38 & & & & 1.30 & & & \\
\hline Lobophora variegata & 0.18 & 0.38 & & & 5.48 & 3.34 & & \\
\hline Padina pavonica & 22.89 & & & & 6.47 & & & \\
\hline Sphacelaria cirrosa & & & & & 0.45 & & & \\
\hline Taonia atomaria & 0.93 & 0.05 & & & & & & \\
\hline Unid. filamentous brown algae & & 0.12 & & & & & & \\
\hline \multicolumn{9}{|l|}{ Rhodophyta } \\
\hline Amphiroa cryptarthrodia & & 0.06 & & & 0.14 & 0.10 & & \\
\hline Amphiroa rigida & 0.91 & & & & 2.88 & & & \\
\hline Boergeseniella fruticulosa & 0.10 & & & & 0.11 & & & \\
\hline Botryocladia boergesenii & & 0.004 & & & & 0.01 & & \\
\hline Botryocladia botryoides & & 0.004 & & & 0.09 & & & \\
\hline Contarinia squamariae & & 0.12 & & & & 0.07 & & \\
\hline Corallina elongata & 0.40 & & & & 0.43 & 0.003 & & \\
\hline Cryptonemia lomation & & & & & 0.004 & & & \\
\hline Delesseriaceae unid. & & 0.12 & & & & 0.53 & & \\
\hline Falkenbergia sp. & 0.61 & 0.09 & & & 0.22 & & & \\
\hline Gloiocladia furcata & & 0.10 & & & 0.004 & 0.31 & & \\
\hline Haliptilon virgatum & 9.19 & 0.25 & & & 36.95 & 0.46 & & \\
\hline Hydrolithon farinosum & & 1.83 & 0.004 & & & 3.77 & 0.004 & \\
\hline Laurencia gr. obtusa & & & & & 0.002 & & & \\
\hline Lithophyllum cabiochae & & 0.27 & & & & & & \\
\hline Melobesiae unid. & & & 0.64 & & & 0.24 & 0.89 & \\
\hline Mesophyllum alternans & 0.49 & 7.95 & & & 2.11 & 3.08 & & \\
\hline Neogoniolithon brassica-florida & 7.44 & & & & 28.59 & & & \\
\hline Peyssonnelia rosa-marina & & 32.31 & & & & 44.90 & & \\
\hline Peyssonnelia squamaria & 0.30 & 13.38 & & & 0.30 & 9.47 & & \\
\hline Plocamium cartilagineum & & 0.19 & & & & 0.02 & & \\
\hline Polystrata fosliei & & & & & 1.80 & 0.05 & & \\
\hline Rhodymenia ardissonei & & & & & & 0.004 & & \\
\hline Tricleocarpa sp. & & 0.001 & & & 0.08 & 0.01 & & \\
\hline Unid. filamentous red algae & & 2.37 & & & & & & \\
\hline Wurdermannia miniata & & & & & 1.86 & & & \\
\hline \multicolumn{9}{|l|}{ Granuloreticulosa } \\
\hline Miniacina miniacea & & 0.04 & 0.08 & 0.47 & & & 0.12 & 0.12 \\
\hline \multicolumn{9}{|l|}{ Porifera } \\
\hline Acanthella acuta & & 0.20 & 0.06 & & & 0.15 & 0.10 & \\
\hline * Agelas oroides & & & 1.29 & 1.61 & & & 0.98 & 1.61 \\
\hline * Axinella damicornis & & 0.60 & 1.49 & 1.39 & & 0.48 & 2.63 & 1.39 \\
\hline Cacospongia mollior & & 0.08 & & & & & & \\
\hline Chondrosia reniformis & & 0.03 & 1.92 & 0.001 & & & 1.51 & 0.004 \\
\hline
\end{tabular}


Table 4. (Continued).

\begin{tabular}{|c|c|c|c|c|c|c|c|c|}
\hline \multirow{2}{*}{$\begin{array}{l}\text { Species } \\
\text { Cabrera cave }\end{array}$} & \multicolumn{4}{|c|}{ June } & \multicolumn{4}{|c|}{ November } \\
\hline & Zone 1 & Zone 2 & Zone 3 & Zone 4 & Zone 1 & Zone 2 & Zone 3 & Zone 4 \\
\hline Clathrina clathrus & & 0.01 & & 0.003 & & 0.004 & & \\
\hline Clathrina sp. & & & & 0.01 & & & & \\
\hline Cliona celata & & & & & 0.03 & & & \\
\hline Cliona sp. & 0.01 & 0.18 & & & & & & \\
\hline Cliona viridis & 0.01 & & & & & & & \\
\hline * Crambe crambe & 0.46 & 1.13 & 0.01 & 1.05 & 0.03 & 0.18 & 0.02 & 1.05 \\
\hline * Darwinella sp. & & & & 0.01 & & & & 0.01 \\
\hline * Dendroxea lenis & & & 1.37 & 5.59 & & & 0.71 & 5.59 \\
\hline * Diplastrella bistellata & & & 0.40 & 6.66 & & & 0.27 & 6.66 \\
\hline * Dictyonella sp. & & & 2.49 & 0.85 & & & 0.75 & 0.85 \\
\hline * Dysidea avara & & 0.09 & & 0.63 & & 0.07 & & 0.63 \\
\hline Erylus euastrum & & 0.14 & 3.84 & 1.24 & & 0.20 & 0.91 & 2.78 \\
\hline Eurypon sp. & & & 0.17 & 0.43 & & & 0.28 & 0.32 \\
\hline Hexadella pruvotii & & & 0.03 & 0.64 & & & 0.06 & 1.07 \\
\hline Hippospongia communis & & & 0.01 & & & & 0.01 & 0.18 \\
\hline *Hymedesmia sp. 1 & & & 0.89 & 1.01 & & & 0.18 & 1.01 \\
\hline *Hymedesmia sp. 2 & 0.14 & & & 1.75 & & & & 1.75 \\
\hline *Hymedesmia sp. 4 & & & & 10.62 & & & & 10.62 \\
\hline Ircinia fasciculata & & & & & & & 0.21 & \\
\hline Ircinia oros & & & 0.82 & & & & 0.53 & \\
\hline Ircinia variabilis & & & 1.26 & 0.45 & & 0.04 & 4.88 & 1.30 \\
\hline Leucosolenia variabilis & & & 0.001 & 0.01 & & & & 0.001 \\
\hline Microciona cf. ascendens & & & 0.01 & & & & & \\
\hline *Microciona sp. & & & & 0.46 & & & & 0.46 \\
\hline Myceliospongia araneosa & & & 0.14 & 0.40 & & & 0.30 & 0.26 \\
\hline Oscarella tuberculata & & & 0.87 & 0.11 & & & 0.92 & 0.21 \\
\hline * Petrosia ficiformis & & & & 0.04 & & & & 0.04 \\
\hline Phorbas fictitius & & 0.07 & 0.07 & & 0.22 & & 0.09 & \\
\hline Phorbas tenacior & & 0.06 & 2.34 & 1.43 & & & 2.21 & 3.22 \\
\hline Pione vastifica & 0.001 & & & & & & & \\
\hline Pleraplysilla spinifera & & 0.32 & 1.47 & 0.34 & & 0.20 & 2.30 & 0.25 \\
\hline Raspaciona aculeata & & & 0.48 & 0.21 & & & 0.85 & 0.46 \\
\hline Reniera fulva & & & 0.02 & 0.002 & & & & \\
\hline Reniera mucosa & & 0.19 & & 0.03 & & & & 0.01 \\
\hline * Spirastrella cunctatrix & & & 4.62 & 6.44 & & & 3.12 & 6.44 \\
\hline Spongia virgultosa & & & 0.16 & & 0.01 & & & \\
\hline Terpios fugax & & 0.01 & 0.04 & 0.01 & & 0.02 & 0.01 & 0.03 \\
\hline Topsentia garciae & & & 0.24 & 0.10 & & & 0.35 & 0.34 \\
\hline \multicolumn{9}{|l|}{ Cnidaria } \\
\hline Aglaophenia sp. & 0.12 & & & & 0.08 & & & \\
\hline Balanophyllia europaea & 0.11 & & & & 0.20 & & & 0.01 \\
\hline Caryophyllia inornata & & 0.20 & 0.003 & & & 0.17 & & \\
\hline Clavularia crassa & & 0.36 & & & 0.12 & 0.43 & & \\
\hline Cornularia cornucopiae & & 0.01 & & & & 0.25 & & \\
\hline Eudendrium sp. & & 0.47 & & & & 0.01 & & \\
\hline Hydrozoan unid. & & & & & & 0.27 & & \\
\hline Hoplangia durotrix & & 0.06 & & 0.42 & & & & 0.06 \\
\hline Leptopsammia pruvoti & & 1.78 & 0.06 & 0.26 & 0.03 & 0.61 & 0.01 & 0.17 \\
\hline Maasella edwardsi & & & & & 0.06 & & & \\
\hline Parerythropodium coralloides & & & & & & & & 0.00 \\
\hline Parazoanthus axinellae & & & 9.33 & 1.21 & & & 13.85 & 2.57 \\
\hline Polycyathus muellerae & & 3.55 & 0.10 & 0.43 & & 3.14 & 0.73 & 1.11 \\
\hline \multicolumn{9}{|l|}{ Polychaeta } \\
\hline Protula sp. & & 0.09 & 0.87 & 0.003 & & 0.03 & 0.07 & \\
\hline Salmacina dysteri & & & & 0.02 & & & & \\
\hline Serpula vermicularis & 0.40 & & 0.01 & 0.002 & & & & 0.68 \\
\hline Serpulidae sp. 1 & & & & 0.01 & & & & \\
\hline Serpulidae sp. 2 & & & 0.43 & 0.24 & & & 0.21 & 0.08 \\
\hline Serpulidae sp. 3 & & & 0.05 & 1.09 & & & & 0.73 \\
\hline Serpulidae sp. 4 & & & 0.02 & 0.20 & & & 0.01 & \\
\hline
\end{tabular}


Bryozoa

Annectocyma indistincta

Annectocyma sp.

Bryozoan sp. 1

Bryozoan sp. 2

Bryozoan sp.3

Bryozoan sp.4

Bryozoan sp. 5

Bryozoan sp. 6

Bryozoan sp. 7

Bryozoan sp.8

Bugula calathus

Cellaria sp.

Celleporina sp.

Chlidonia pyriformis

Crisia sp. 1

Crisia sp. 2

Crisia sp.3

Crisia sp. 4

Crisia sp. 5

Crisia sp. 6

Frondipora verrucosa

Lichenopora radiata

Margaretta cereoides

Myriapora truncata

Puellina gattyae

Reptadeonella violacea

Rynchozoon sp.

Schizomavella sp. 1

Schizomavella sp. 2

Schizomavella sp.3

Scrupocellaria sp.

Sertella sp.

Smittina cervicornis

Mollusca

Bittium reticulatum

Calliostoma sp.

Lima hians

Lithophaga lithophaga

Patella sp.

Brachiopoda

Argyrotheca cordata

Echinodermata

Paracentrotus lividus

Tunicata

Ascidia mentula

Didemnum sp. 1

Didemnum sp. 2

Lissoclinum perforatum

Pyura dura

Unidentified

Bare rock
0.10

$\begin{array}{lllll}0.002 & & 0.01 & 0.004 & \\ 0.08 & 0.05 & 0.02 & 0.02 & 0.02 \\ 0.05 & & & & \\ & & 0.33 & & \\ 0.01 & 0.33 & & 0.01 & 0.19 \\ & & & & 0.01\end{array}$

0.13

0.01

1.19

0.01

0.36

0.001

0.01

0.01

0.02

0.04

0.39

0.07

1.48

0.16

0.32

0.003

0.03

0.39

0.58

0.01

0.64

0.71

$0.03 \quad 0.04$

0.05

0.02

4.74

0.09

0.12

0.01

3.16

0.30

0.12

0.31

5.37

1.94

$0.01 \quad 0.89$

0.34

0.42

0.55

$1.21 \quad 5.97$

5.97
0.56

0.06

5.41

1.12

3.86

6.84

0.44

0.003

0.03

0.01

0.05

0.03

0.01

0.03

0.14

0.03

0.01

0.01

0.01

0.14

0.01

0.02

0.03

$0.11 \quad 0.08$

0.04

0.01

0.02

$0.01 \quad 0.04$

0.01

0.07

0.13

$\begin{array}{lll}1.48 & 5.27 & 7.66\end{array}$

0.95

0.20

6.43

10.42

0.74

$0.06 \quad 37.23$

46.05

0.10

0.10

0.43
Botrylloides leachii

\begin{tabular}{|c|c|c|c|c|c|c|c|c|}
\hline \multirow{2}{*}{$\begin{array}{l}\text { Species } \\
\text { Medes cave }\end{array}$} & \multicolumn{4}{|c|}{ June } & \multicolumn{4}{|c|}{ November } \\
\hline & Zone 1 & Zone 2 & Zone 3 & Zone 4 & Zone 1 & Zone 2 & Zone 3 & Zone 4 \\
\hline \multicolumn{9}{|l|}{ Chlorophyta } \\
\hline Cladophora pellucida & 0.02 & & & & 0.04 & & & \\
\hline Cladophora sp. & 0.11 & & & & 1.33 & & & \\
\hline Flabellia petiolata & 0.56 & & & & 2.27 & & & \\
\hline Halicystis paroula & 0.001 & 0.001 & & & & & & \\
\hline Halimeda tuna & 0.07 & & & & 0.06 & & & \\
\hline Pseudochlorodesmis furcellata & 0.003 & & & & & & & \\
\hline
\end{tabular}


Table 4. (Continued).

\begin{tabular}{|c|c|c|c|c|c|c|c|c|}
\hline \multirow{2}{*}{$\begin{array}{l}\text { Species } \\
\text { Medes cave }\end{array}$} & \multicolumn{4}{|c|}{ June } & \multicolumn{4}{|c|}{ November } \\
\hline & Zone 1 & Zone 2 & Zone 3 & Zone 4 & Zone 1 & Zone 2 & Zone 3 & Zone 4 \\
\hline \multicolumn{9}{|l|}{ Phaeophyta } \\
\hline Aglaozonia sp. & 0.28 & & & & 0.24 & & & \\
\hline Colpomenia sinuosa & 0.12 & & & & & & & \\
\hline Dictyopteris polypodioides & & & & & 0.02 & & & \\
\hline Dictyota dichotoma & 1.05 & & & & 0.06 & & & \\
\hline Dictyota dichotoma v. intricata & 5.27 & & & & & & & \\
\hline Dictyota fasciola & 1.81 & & & & 0.04 & & & \\
\hline Halopteris filicina & 1.36 & & & & 3.41 & & & \\
\hline Halopteris scoparia & 1.35 & & & & & & & \\
\hline Padina pavonica & 0.01 & & & & & & & \\
\hline Taonia atomaria & 24.57 & & & & & & & \\
\hline \multicolumn{9}{|l|}{ Rhodophyta } \\
\hline Amphiroa beauvoisii & & & & & 0.05 & & & \\
\hline Amphiroa cryptarthrodia & 0.01 & & & & 0.14 & & & \\
\hline Asparagopsis armata & 3.57 & & & & & & & \\
\hline Bornetia secundiflora & 0.01 & & & & & & & \\
\hline Corallina elongata & 2.37 & & & & 11.27 & & & \\
\hline Falkenbergia rufolanosa & 33.35 & & & & 45.20 & & & \\
\hline Hydrolithon farinosum & 0.25 & & & & & & & \\
\hline fania rubens & 0.08 & & & & 0.09 & & & \\
\hline Lithophyllum dentatum & 0.13 & & & & & & & \\
\hline Lithophyllum incrustans & 1.78 & & & & 1.47 & & & \\
\hline Melobesiae unid. & & 0.03 & & & & 0.02 & & \\
\hline Mesophyllum alternans & 2.65 & & & & 11.35 & & & \\
\hline Peyssonnelia rosa-marina & 0.20 & & & & 1.47 & & & \\
\hline Peyssonnelia squamaria & 0.81 & & & & 3.34 & & & \\
\hline Plocamium cartilagineum & 0.52 & & & & 0.44 & & & \\
\hline Rhodophyllis divaricata & 0.003 & & & & & & & \\
\hline Rhodymenia ardissonei & & 0.20 & & & & & & \\
\hline Unid. filamentous red algae & & & & & 8.65 & & & \\
\hline \multicolumn{9}{|l|}{ Granuloreticulosa } \\
\hline Miniacina miniacea & & 0.001 & & & & & & 0.14 \\
\hline \multicolumn{9}{|l|}{ Porifera } \\
\hline Aaptos aaptos & & 0.04 & 0.72 & 0.01 & & 0.41 & 0.12 & 0.45 \\
\hline Acanthella acuta & & 0.38 & & & & 0.07 & & \\
\hline Agelas oroides & & 1.67 & 0.20 & 0.04 & & 2.50 & 1.54 & 0.03 \\
\hline Aplysina cavernicola & & 0.05 & 1.62 & 0.53 & & 0.41 & 2.06 & 0.75 \\
\hline Axinella damicornis & & 0.01 & & & & 0.30 & & 0.03 \\
\hline Cacospongia mollior & & & & & 0.01 & & & \\
\hline Chelanoplysilla noevus & & 0.004 & 0.001 & & & 0.14 & 0.004 & \\
\hline Chondrosia reniformis & & 0.003 & & 0.01 & & & 0.01 & 0.03 \\
\hline Clathrina sp. 1 & 0.001 & 0.06 & 0.29 & 0.90 & 0.05 & 0.09 & 0.40 & 1.02 \\
\hline Clathrina clathrus & & 2.48 & 2.40 & 0.57 & 0.001 & 1.90 & 1.28 & 0.70 \\
\hline Clathrina sp. 2 & & & & 0.01 & & & & \\
\hline Cliona schmidti & & & & 0.01 & & & 0.001 & 0.001 \\
\hline Clionia celata & 0.01 & & & & 0.01 & & & \\
\hline Clionia viridis & 0.01 & 0.003 & & & 0.01 & & 0.06 & \\
\hline Crambe crambe & 0.98 & & & & 1.90 & 0.001 & & \\
\hline Crella mollior & & 0.05 & 0.18 & 0.08 & & 0.10 & 0.11 & 0.22 \\
\hline Dendroxea lenis & 0.01 & 11.49 & 15.71 & 7.70 & 0.01 & 7.49 & 11.48 & 12.52 \\
\hline Diplastrella bistellata & & 8.57 & 9.34 & 0.89 & & 10.14 & 10.70 & 3.30 \\
\hline Dictyonella sp. & & 0.23 & & & & 1.09 & & 0.07 \\
\hline Dysidea avara & & 1.05 & 0.75 & & & 1.76 & 0.46 & 0.003 \\
\hline Erylus euastrum & & 0.07 & 0.20 & 0.92 & & 0.15 & 1.31 & 2.38 \\
\hline Eurypon sp.1 & & 0.28 & & & 0.26 & 0.09 & & \\
\hline Eurypon sp.2 & & & 0.03 & & & & & \\
\hline Hemimycale columella & & & & & 0.01 & & & \\
\hline Hexadella pruvotii & & 0.002 & 0.01 & 0.02 & & 0.39 & 0.002 & \\
\hline Hymedesmia sp. 1 & & 0.07 & 0.06 & 0.85 & & 0.27 & 0.11 & 2.46 \\
\hline Hymedesmia sp. 2 & & & 0.13 & 0.06 & & & 0.06 & \\
\hline \multirow[t]{2}{*}{ Hymedesmia sp. 4} & & & 0.70 & 0.003 & & 0.24 & & 0.01 \\
\hline & & & & & \multicolumn{4}{|c|}{0.32} \\
\hline
\end{tabular}


Ircinia fasciculata

Ircinia oros

Ircinia variabilis

Leucosolenia variabilis

Microciona sp.

Mycale sp.

Myceliospongia araneosa

Oscarella sp.

Oscarella tuberculata

Petrosia ficiformis

Phorbas fictitius

Phorbas tenacior

Pione vastifica

Pleraplysilla spinifera

Reniera mucosa

Reniera sarai

Reniera sp.

Spirastrella cunctatrix

Spongia virgultosa

Sycon elegans

Sycon sp.

Terpios fugax

Topsentia garciae

Cnidaria

Aglaophenia kirchenpaueri

Alcyonium acaule

Balanophyllia regia

Caryophyllia inornata

Corallium rubrum

Cornularia cornucopiae

Eudendrium sp.

Eunicella singularis

Hydrozoan unid.

Hoplangia durotrix

Leptopsammia pruvoti

Polycyathus muellerae

Sertularella ellisi

Sertularella sp.

Polychaeta

Myxicola aesthetica

Protula sp.

Salmacina dysteri

Serpula vermicularis

Serpulidae sp. 1

Serpulidae sp. 2

Serpulidae sp. 3

Serpulidae sp. 4

Serpulidae sp. 5

Spirorbis sp.

Bryozoa

Bryozoan sp. 1

Bryozoan sp. 2

Bryozoan sp. 3

Bryozoan sp. 4

Celleporina sp.

Chlidonia pyriformis

Crisia sp.

Disporella hispida

Frondipora verrucosa

Idmidronea atlantica

Lichenopora radiata

Myriapora truncata

Parasmittina tropica

Schizomavella cuspidata

Schizomavella linearis

Schizomavella sp. 1

Schizomavella sp. 2
0.18

0.00

(1)

(20)

0.04

0.02

0.01

.

0.01

5.69

1.08

0.003

0.58

0.07

0.26

0.30

$\begin{array}{lll}0.34 & 0.03 & 0.26 \\ 0.36 & 1.46 & 1.51\end{array}$

0.001

0.50

0.17

0.08

0.08

0.04

0.01

0.002

0.002

0.04

0.35

0.12

0.12

0.02

6.57

3.29

0.001

6.19

2.57

0.02

0.03

0.02

2.85

1.80

2.13

0.89

0.04

1.00

0.39

3.75

0.003

5.53

0.004

0.002

2.60

0.01

0.01

0.02

0.02

0.13

0.03

0.01

0.01

0.02

0.02

0.00

0.03

0.01

0.06

0.47

0.002

0.09

0.01

0.04

0.19

0.02

0.003

0.02

1.73

0.09

0.02

0.42

0.004

0.09

0.06

0.10

0.01

0.00

0.04

0.02

$0.03 \quad 0.07$

0.01

0.34

0.06

0.16

0.03

0.14

0.01

0.11

0.01

0.47

4.35

0.07

0.01

0.03

0.37

0.03

0.02

0.09

0.32

3.63

0.05

0.06

0.01

0.01

0.001

0.03

0.01

0.15

0.06

0.04

0.04

0.001

0.002

0.01

0.14

0.73

0.06

0.01

0.01
0.07

0.01

0.03

1.07

1.23
0.19

1.05

0.16

0.001

0.01

0.003

0.17

0.001

0.02

0.33

0.05

0.20

3.05

0.001

0.09

2.02

0.01

0.01

0.2

0.40

0.73

0.05

0.003

0.002

0.70 
Table 4. (Continued).

\begin{tabular}{|c|c|c|c|c|c|c|c|c|}
\hline \multirow{2}{*}{$\frac{\text { Species }}{\text { Medes cave }}$} & \multicolumn{4}{|c|}{ June } & \multicolumn{4}{|c|}{ November } \\
\hline & Zone 1 & Zone 2 & Zone 3 & Zone 4 & Zone 1 & Zone 2 & Zone 3 & Zone 4 \\
\hline \multicolumn{9}{|l|}{ Bryozoa (continued) } \\
\hline Schizomavella sp. 3 & & & 0.04 & & & & 0.10 & \\
\hline Scrupocellaria sp. & & 5.35 & 1.31 & 0.04 & & 0.60 & 0.22 & 0.05 \\
\hline Sertella sp. & 0.02 & & & & 0.02 & 0.01 & & \\
\hline Smittina cervicornis & & 0.59 & & & & 0.31 & & \\
\hline Smittoidea reticulata & & 0.20 & & & & 0.01 & & \\
\hline Spiralaria gregaria & & 0.09 & & & & 0.85 & & 0.40 \\
\hline \multicolumn{9}{|l|}{ Mollusca } \\
\hline Bittium reticulatum & & & & & & & & 0.001 \\
\hline Bivalvia unid. & & & 0.003 & & & & & \\
\hline Coralliophila sp. & & & 0.001 & & & & 0.003 & \\
\hline Lithophaga lithophaga & 2.35 & & 0.01 & & 0.03 & & 0.01 & \\
\hline \multicolumn{9}{|l|}{ Brachiopoda } \\
\hline Argyrotheca cordata & & & & & & & 0.002 & 0.001 \\
\hline Terebratulina sp. & & & 0.01 & 0.02 & & & 0.04 & \\
\hline Crania anomala & & & 0.15 & 2.31 & & & 0.12 & 0.91 \\
\hline Megerlia truncata & & & 0.001 & 0.01 & & 0.003 & 0.001 & 0.02 \\
\hline \multicolumn{9}{|l|}{ Echinodermata } \\
\hline Paracentrotus lividus & 0.42 & & & & & 0.01 & & \\
\hline \multicolumn{9}{|l|}{ Tunicata } \\
\hline Clavelina lepadiformis & 0.10 & & & & 0.54 & & & \\
\hline Cystodytes dellechiajei & 0.08 & 0.12 & 0.01 & & 0.11 & 0.42 & 0.001 & \\
\hline Didemnum sp. 1 & 0.01 & 0.05 & 0.36 & 0.61 & & 0.04 & 0.18 & 0.94 \\
\hline Didemnum sp. 2 & 0.01 & & & & & & & \\
\hline Didemnum sp. 3 & & & 0.03 & & & & 0.004 & 0.33 \\
\hline Didemnum sp. 4 & & & & & & & 0.004 & \\
\hline Diplosoma spongiforme & & & 0.04 & & & & & \\
\hline Pyura dura & & 0.02 & & & & & & \\
\hline Unidentified & 13.21 & 2.35 & 1.26 & 0.12 & 5.23 & 0.39 & 1.95 & 1.18 \\
\hline Bare rock & & 35.84 & 48.28 & 73.20 & & 40.56 & 51.55 & 60.69 \\
\hline
\end{tabular}

Note that in Zone 4 of the Cabrera cave some sponge species $(*)$ have exactly the same coverage in June and November. Although these species in fact decreased in coverage, we consider this decrease an artefact due to the small size of the zone and the intensive sampling for toxicity analyses carried out in June. Some sponge specimens that remained untouched showed an increment in coverage from June to November. We have kept the coverage values of these species for June, as we think that this is the more conservative correction we can apply.

polychaetes were the groups best represented after the sponges.

There were marked seasonal differences in species richness (Figure 3). In the Cabrera cave there was a noticeable increase in the number of species in Zone 1 from June to November, and a decrease in Zones 2, 3 and 4 during the same period. In the Medes cave there was a decrease in the number of species from June to November in Zones 1 and 2, while Zones 3 and 4 showed an increase in the number of species in the same period.

\section{Ordination}

The ordination analysis of the samples from the Cabrera cave is graphically represented in Figure 4A,B. The first axis was interpreted as representing the horizontal gradient across the cave from the most external zone (left in the graph) to the innermost zone (right in the graph). Forty-seven per cent of the variation of our pictures was explained by the two first axes. However, almost all the variance $(77.6 \%)$ was explained by axis I. Notice that the $r^{2}$ for axis II is low in all the graphs (Figure 4).
The 20 pictures taken at the most external part of the cave (zone 1), appeared in the analysis as a compact group on the left part of the graph. Clearly separated from the first group, another cluster of 20 samples, which belonged to Zone 2, were represented.

The set of samples on the right of the graph belonged to Zones 3 and 4 . These two zones were situated inside the cave, with low levels of light, so the dominant benthic organisms there were invertebrates. No spatial separation of the two zones was obtained in this analysis.

In order to assess whether the lack of resolution between the two internal zones (Zones 3 and 4) was an artefact due to the presence of the other, markedly different zones, another analysis was carried out with only the 40 pictures of these two zones (Figure 4B). After the elimination of the outliers, axis I represented the gradient from Zone 3 to the innermost part of the cave (Zone 4). Most of the samples of Zone 4 can be found on the right part of the graph. Axis I explained $37 \%$ of the variance in the samples.

Most of the samples from Zone 3 were grouped on the left. However, the separation of the two zones was not clear cut. This was expected since the species found were 
Cabrera cave

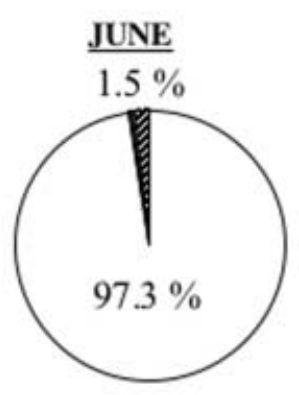

NOVEMBER

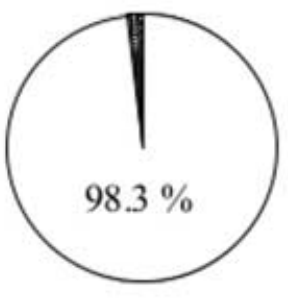

Zone 1

$1.3 \%$

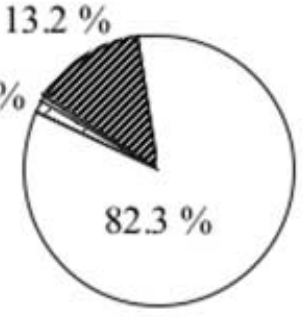

NOVEMBER

$2.6 \% \quad 5.2 \%$

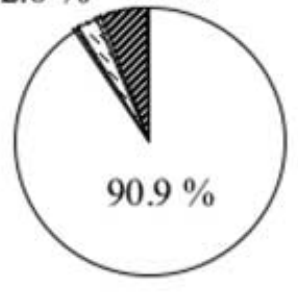

Zone 2
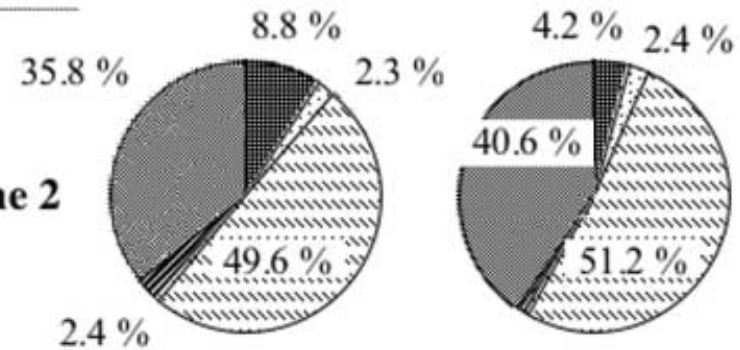

$1.2 \% 1 \%$
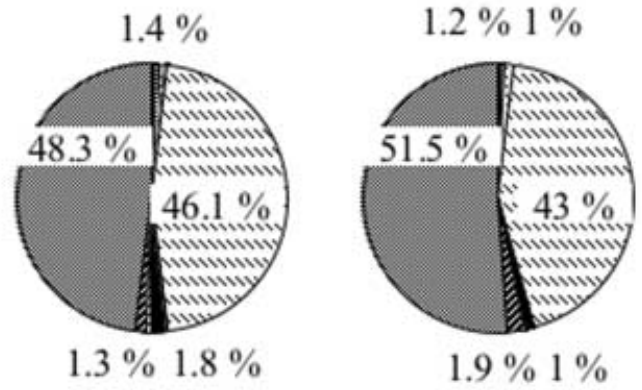
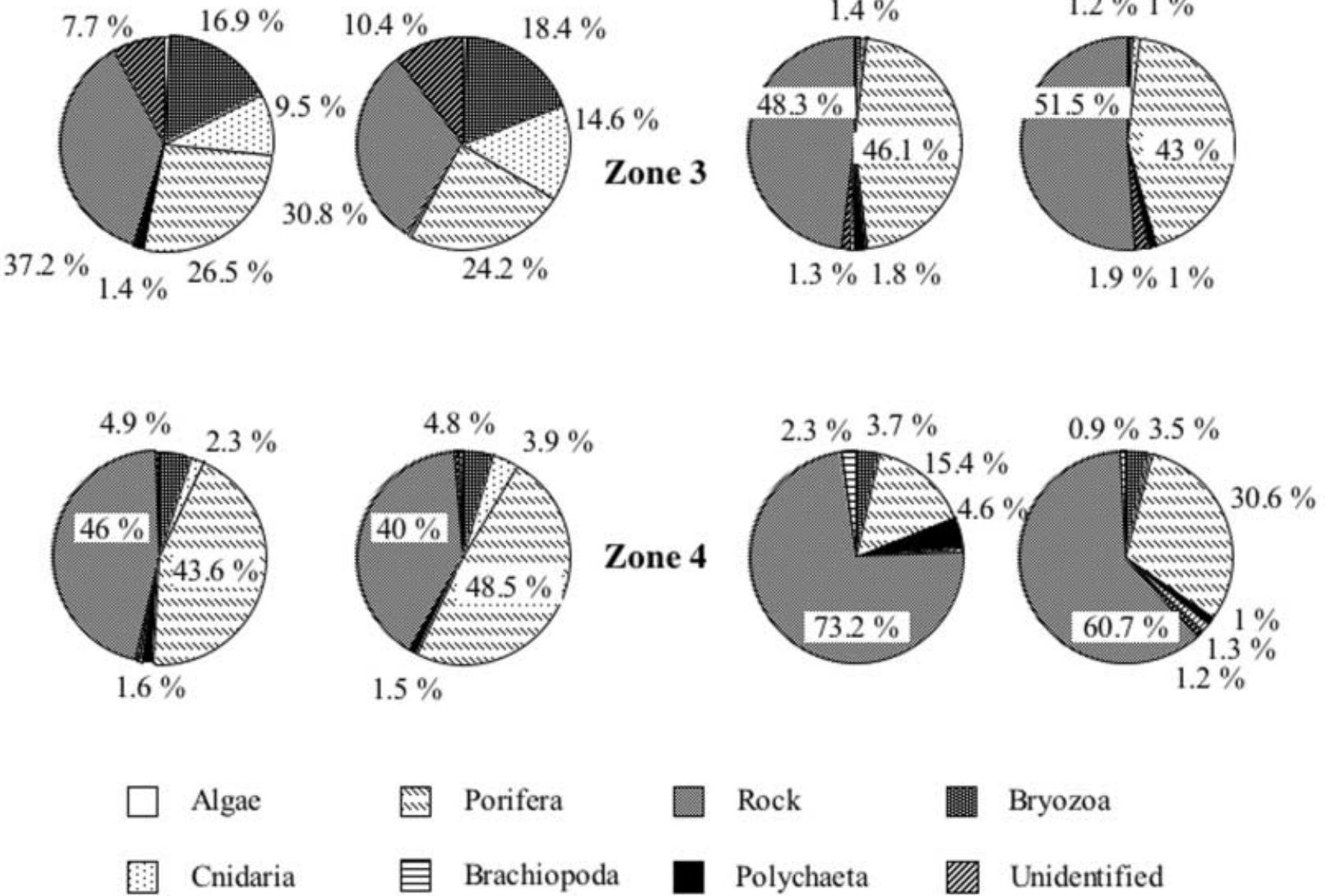

Rock

Bryozoa

目 Brachiopoda

Polychaeta

Unidentified

Figure 2. Coverage per high-level taxonomic group in the four zones of the Cabrera cave and the Medes cave in both seasons. 
Cabrera cave

JUNE
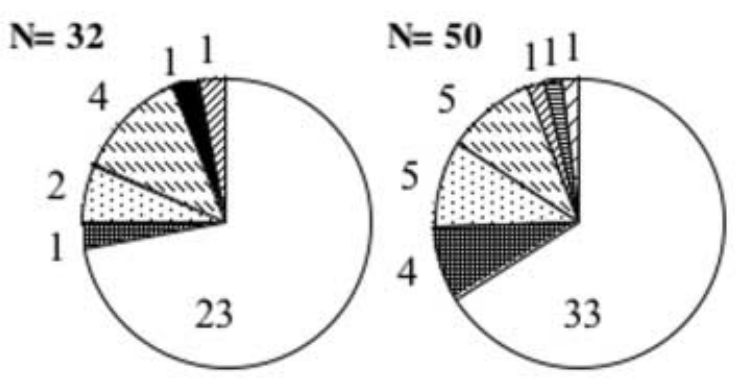

$\mathrm{N}=70$
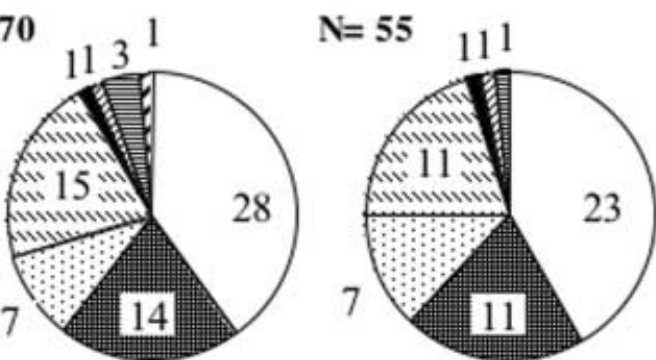

$\mathrm{N}=55$

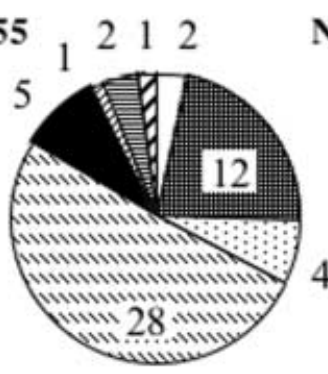

$\mathrm{N}=49_{3} \quad 1 \quad 1 \quad 2$

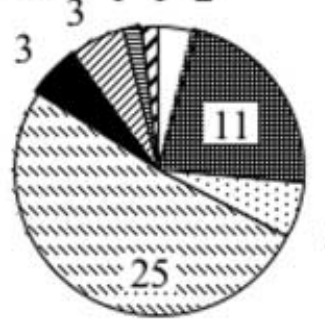

Zone 2

$\mathrm{N}=56$

Zone 1
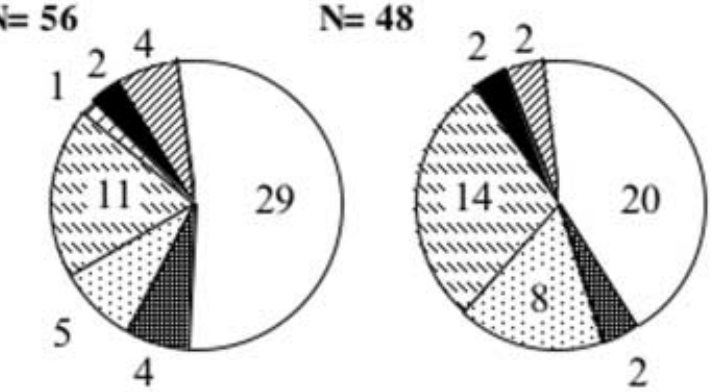

Medes cave

JUNE

NOVEMBER

Zone 3

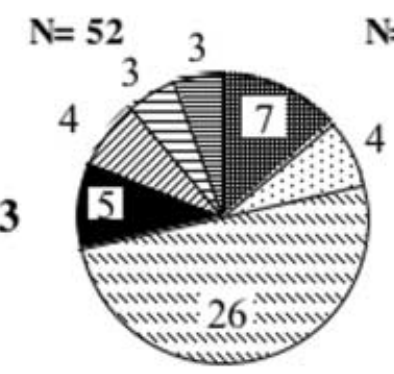

$\mathrm{N}=\mathbf{5 8}$

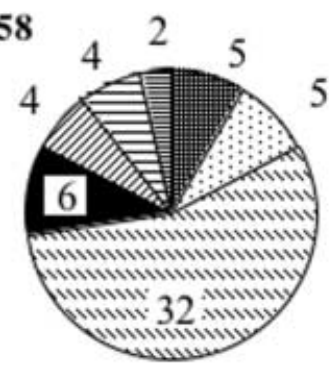

$\mathrm{N}=64$

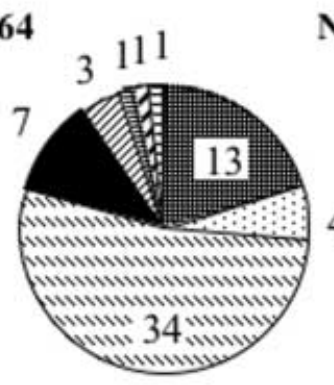

$\mathbf{N}=\mathbf{5 8}$

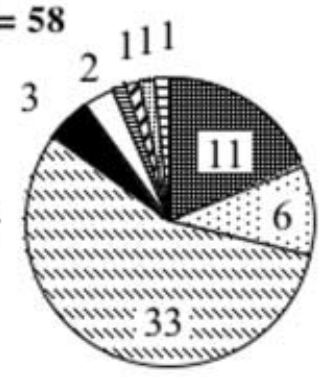

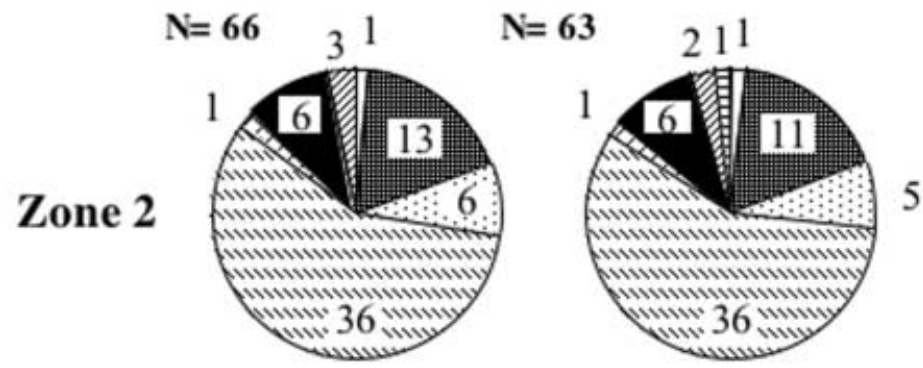

$\mathrm{N}=49$

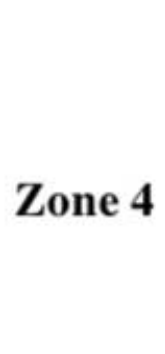

$\mathrm{N}=41$
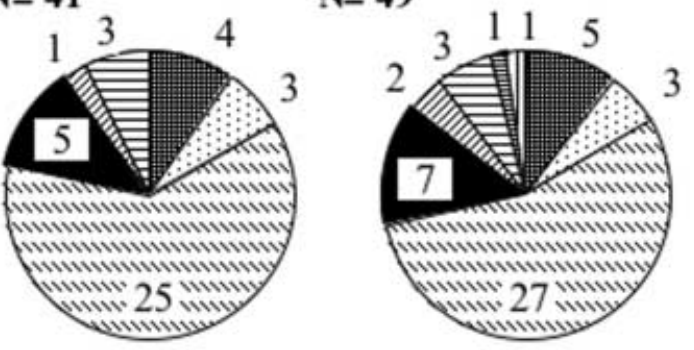

Porifera

Bryozoa

Cnidaria

IIscidiacea

Mollusca

Figure 3. Number of species per high-level taxonomic group in the four zones of the Cabrera cave and the Medes cave in both seasons. Bold values indicate species richness. 
A.

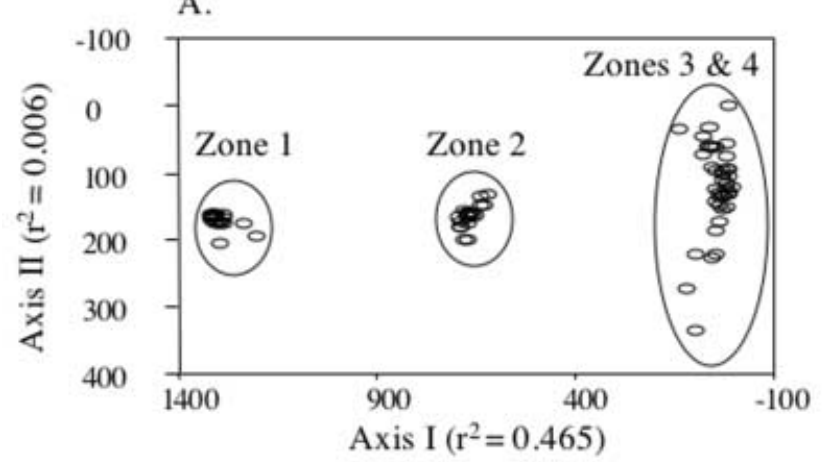

B.
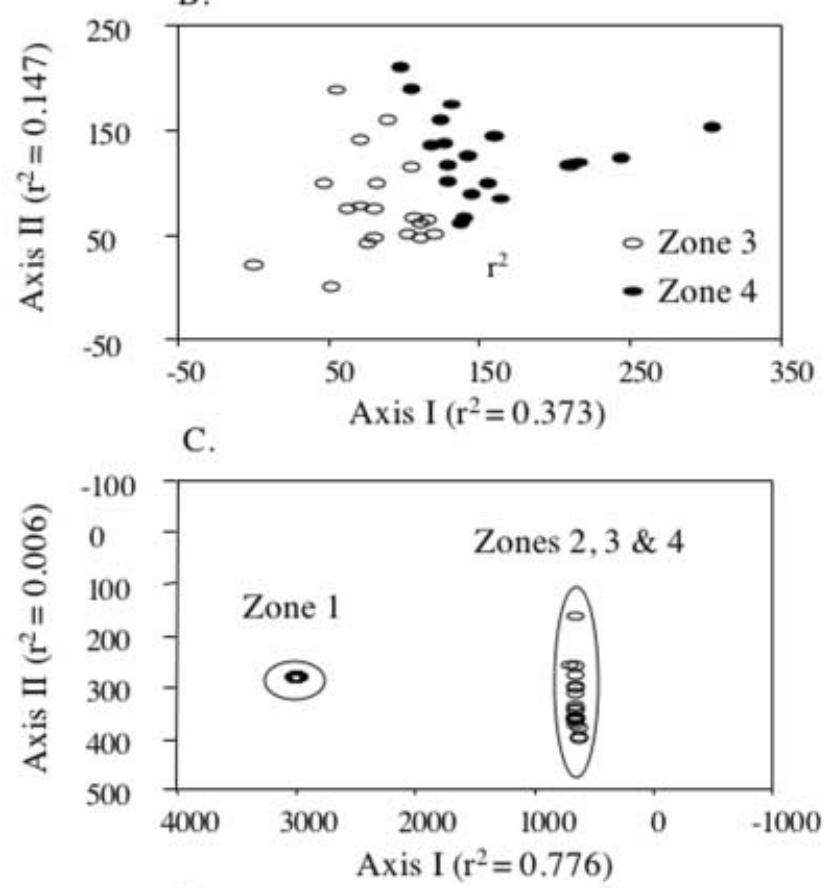

D.

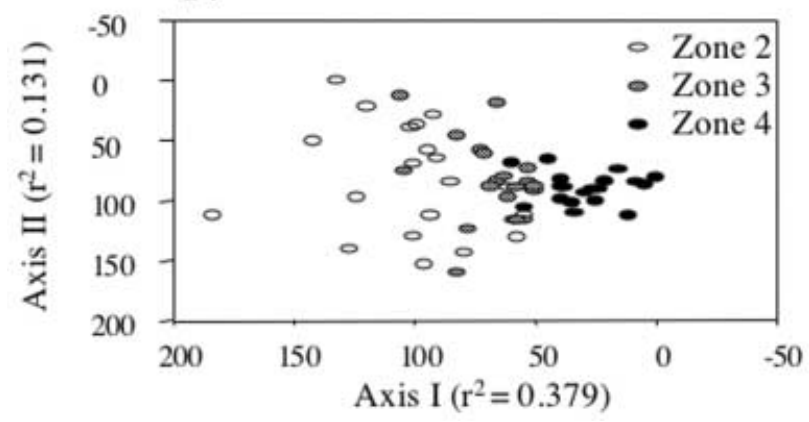

Figure 4. Detrended correspondence analyses (DCAs) representation of all samples (A) and samples of Zones 3 and 4, exclusively (B), from the Cabrera cave and DCAs representation of all samples $(\mathrm{C})$ and samples of Zones 2, 3 and 4, exclusively (D) from the Medes cave. Data represent coverage values in June.

similar in both zones. Axis II distributed samples according to species abundance and amount of bare rock.

The representation of ordination analyses for the Medes cave in June is shown in Figure 4C,D. When all zones were included (Figure 4G), the first axis, which explained $41 \%$ of the total variance, again represented the horizontal gradient along the cave.

The 20 pictures taken in Zone 1 appeared in the analysis in a compact group on the left part of the graph. Clearly
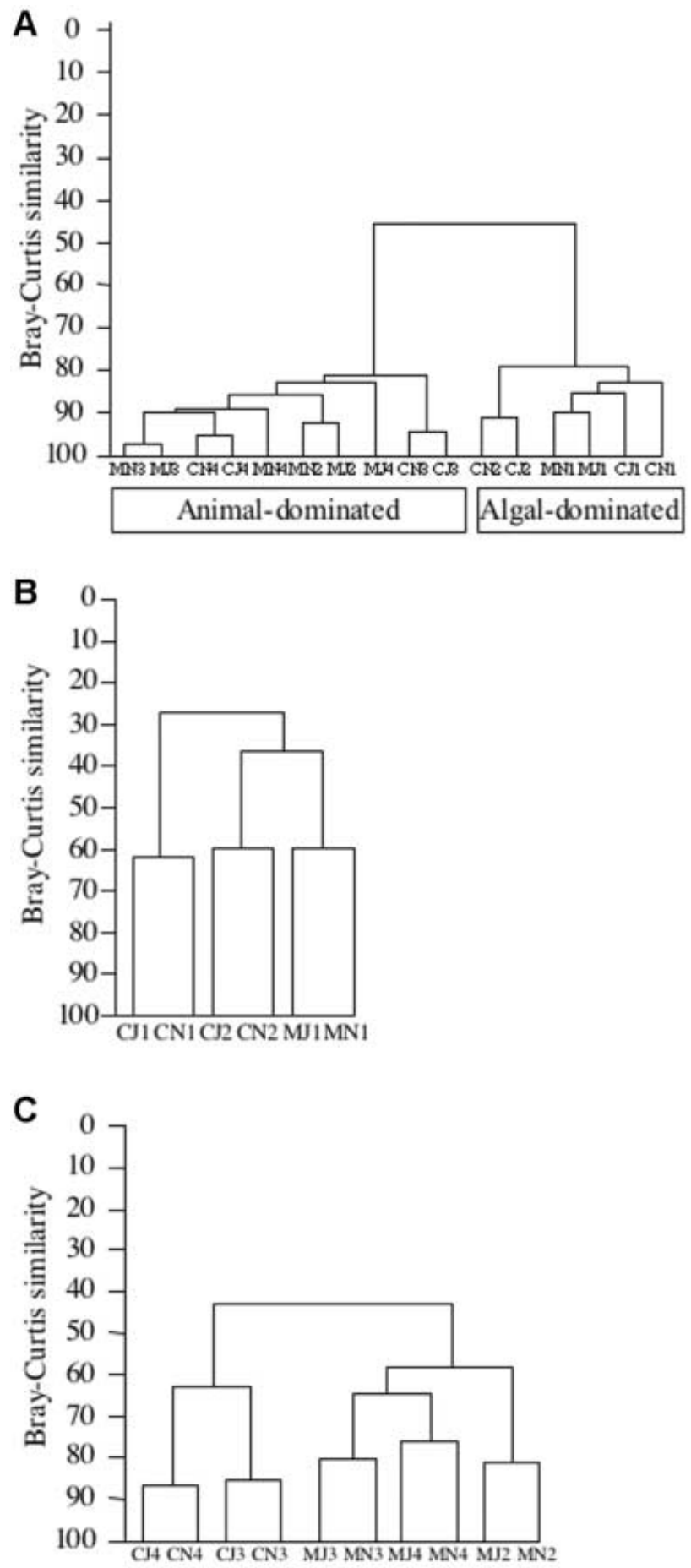

Figure 5. Cluster analysis of zones from both the caves using the percentage cover per high-level taxonomic group (A). Cluster analysis of the algal-dominated zones (B) and animaldominated zones $(\mathrm{C})$, using the percentage cover per species. Data were transformed to 4 th root. C, the Cabrera cave; M, the Medes cave; J, June; N, November. Numbers denote cave zones.

separated from Zone 1, there was a group composed of all the remaining 60 pictures, all of them taken inside the cave.

When the analysis was only performed on the three internal zones (2, 3 and 4) (Figure 4D), axis I again represented the horizontal gradient from the external to the internal zones of the cave, explaining $38 \%$ of the variance. 
The three zones appeared separated along this axis, although with some overlap. This overlap was expected because, as in the Cabrera cave internal zones, the three zones shared the same species. Species abundance and the increment of bare rock were the two factors that clearly varied among zones. Axis II discriminated samples according to the coverage of the most abundant species and bare rock.

\section{Classification}

Two distinct groups were clearly separated in the preliminary cluster analysis performed on high-level taxonomic groups (Figure 5A). The first one comprised all the zones dominated by seaweeds irrespective of cave or season. The second group included all the zones inside the caves (i.e. the darker communities dominated by suspension-feeders). However, relationships within each group were not clear.

A separate analysis on the group comprising the algal-dominated zones (Figure 5B) performed at the species level, showed that Zone 2 of the Cabrera cave was more similar to zone 1 of the Medes cave than to Zone 1 of the Cabrera cave. Thus, two zones belonging to two geographically separated archipelagos were more similar than two zones with spatial continuity. This result highlighted the importance of the amount of light in establishing affinities between different algal communities. Seasonal variation was less important, as the sample of both seasons in each zone clustered together in all cases.

When the animal-dominated communities were analysed at the species level (Figure 5C), the differences in species composition between the two caves became manifest, and the Cabrera and the Medes caves appeared as separate clusters. Again, similarity was higher between seasons for the same zone than between zones.

\section{DISGUSSION}

A clear although different pattern of zonation was found in both the caves. In the Cabrera cave the decrease in coverage and number of species and the corresponding increase in bare rock towards the internal zone is less marked than in the Medes cave (also reported by Gili et al., 1986) possibly due to the higher water flow inside. A diminished larval supply might explain the poor colonization of the substratum at the most internal zones of the caves, since larvae of the main benthic taxa living inside the caves (sponges, bryozoans and ascidians) have not been found in the corresponding planktonic compartment (Palau et al., 1991). However, the difficulty in larval identification of several Phyla (mainly sponges, Mariani et al., 2003) casts some doubt on the absence of larvae in these caves.

Sponges were the dominant group in the animal-dominated communities of both the caves. Bryozoans and cnidarians were also relevant in the Cabrera cave. In contrast, cnidarians were scarce and even absent from the innermost zone in the Medes cave, a finding consistent with the progressive disappearance of cnidarians from semi-dark communities to dark communities previously reported (Harmelin, 1985, 1997).
The adaptation of the organisms from different taxonomic groups to life inside the caves relies mainly on their trophic requirements (Harmelin, 1985). Sponges (active filter-feeders) appear to be well adapted to this kind of environment due to their high efficiency in retention of small organic particulates (Simpson, 1984). As a result, they are highly competitive organisms in submarine caves (Harmelin et al., 1985) and clearly dominate semi-dark communities (Vacelet, 1979). Our data confirmed the reported dominance of sponges in the caves, in terms of both species richness and coverage, from the cave entrance to the innermost zone. However, the pattern of sponge abundance was different in the two caves. While the number of sponge species and their coverage clearly decreased from the external to the internal zone in the Medes cave, both descriptors reached the highest values in the innermost zone (Zone 4) of the Cabrera cave. The differences in species composition (only 38 species out of 62 were present in both the caves) may be due to the geographical isolation of both the caves, and the low dispersal capacity of sponges (Harmelin, 1985; Mariani et al., 2003). When we compare the sponge species composition of the caves studied with that of other Mediterranean caves (e.g. Marseilles caves, Pouliquen, 1972; Harmelin \& Vacelet, 1997; and Migtigliano cave, Balduzzi et al., 1989), we find that the number of species in common is generally low, and decreases with increasing geographic distance, as previously reported (Alcover et al., 1993).

Detrended correspondence analyses revealed differences between the two caves. In all cases the axis with the highest coefficient of determination was the one that represented the horizontal gradient across the cave, from the most external zone to the innermost one.

The cluster analysis on percentage cover per high taxonomic groups allowed us to differentiate those organisms from the external and internal zones of both the caves. The analysis of the external zones (phytobenthic communities) using species percentage cover clustered the zones on the basis of light availability and not by geographic proximity. The internal zones (animal-dominated communities), on the other hand, were grouped according to the cave they belonged to.

All the results presented here show that light is determinant in explaining differences among algal-dominated communities, but not among the animal-dominated communities. The algal communities are similar between the caves, varying as a function of irradiance. In contrast, animal-dominated communities are more similar within than between the caves, and more intrinsic factors, such as cave typology, that determines water flows and hence differences in food and larval supply, may be more relevant to explain differences among animal-dominated benthic communities from the caves. Although seasonal differences in species abundance and coverage are much less important than differences linked to the caves and zones, there is nevertheless significant seasonal variation even in the innermost zones of the caves, especially with respect to coverage figures.

This research was partially supported by INTERREG-IIIA K115C n 0123 (EU) and CICYT (Comision Interdepartmental de Ciencia y Technología) REN2001-2312-CO3/MAR grants. It also benefited from Governments of Catalonia and Spain 
fellowships to R. Martí. We gratefully acknowledge M. Bardají, G. Agell and D. Martin for helping in samples and data processing. We also thank the Cabrera National Park for research facilities.

\section{REFERENCES}

Alcover, J.A., Ballesteros, E. \& Fornós, J.J., 1993. Història natural de l'Arxipèlag de Cabrera, CSIC-Editorial Moll. Monografies de la Societat d'Història Natural de les Balears, 2.

Balduzzi, A., Bianchi, G.N., Boero, F., Cattaneo, R., Pansini, M. \& Sarà, M., 1989. The suspension feeder communities of Mediterranean sea cave. Scientia Marina, 53, 247-250.

Braun Blanquet, J., 1979. Fitosociologia. Bases para el estudio de las comunidades vegetales. Barcelona: Blume Ediciones.

Clarke, K.R. \& Warwick, R.M., 1994. Change in marine communities: an approach to statistical analysis and interpretation. Swindon: Natural Environmental Research Council.

Fichez, R., 1991. Suspended particulate organic matter in Mediterranean submarine cave. Marine Biology, 108, 167-174.

Garrabou, J. \& Flos, J., 1995. A simple diffusion-sedimentation model to explain planktonic gradients within a NW Mediterranean submarine cave. Marine Ecology Progress Series, 123, 273-280.

Gili, J.M., Riera, T. \& Zabala, M., 1986. Physical and biological gradients in a submarine cave on the Western Mediterranean coast (north-east Spain). Marine Biology, 90, 291-297.

Harmelin, J.G., 1985. Organisation spatiale des communautés sessiles des grottes sous-marines de Méditerranée. Rapports et Procès-verbaux des Réunions. Commission Internationale pour l'Exploration Scientifique de la Mer Méditerranée, Paris. 29, 149-153.

Harmelin, J.G., 1997. Diversity of bryozoans in a Mediterranean sublittoral cave with bathyal-like conditions: role of dispersal processes and local factors. Marine Ecology Progress Series, 153, 139-152.

Harmelin, J.G. \& Vacelet, J., 1997. Clues to deep-sea biodiversity in a nearshore cave. Vie Milieu, 47, 351-354.

Harmelin, J.G., Vacelet, J. \& Vasseur, P., 1985. Les grottes sousmarines obscures: un milieu extrême et un remarquable biotope refuge. Tethys, 11, 214-229.

Mariani, S., Uriz, M.J. \& Turon, X., 2003. Methodological bias in the estimations of important meroplanktonic components from near-shore bottoms. Marine Ecology Progress Series, 253, 67-75.

McCune, B. \& Mefford, J.M., 1999. PC-ORD. Multivariate analysis of ecological data, Version 4. Glenden Beach, Oregon, USA: MjM Software Design.

Muus, B.J., 1968. A field method for measuring exposure by means of plaster balls. Sarsia, 34, 61-68.

Palau, M., Cornet, C., Riera, T. \& Zabala, M., 1991. Planktonic gradients along Mediterranean sea cave. Oecologia Aquatica, 10, 299-316.

Pérès, J.M. \& Picard, J., 1964. Nouveau manuel de bionomie Benthique de la Mer Méditerranée. Recueil des Travaux de la Station Marine d'Endoume, 31, 3-137.

Pouliquen, L., 1972. Les spongiaires des grottes sous-marines de la région de Marseille: écologie et systématique. Tethys, 3, $717-758$.

Simpson, T.L., 1984. The cell biology of sponges. New York: SpringerVerlag.

Vacelet, J., 1979. La place des spongiaires dans les systèmes trophiques marins. In Biologie des spongiaires, vol. 291 (ed. C. Lévi and N. Boury-Esnault), pp. 259-270. Paris: Editions du CRNS.

Zabala, M., Riera, T., Gili, J.M., Barangel, M., Lobo, A. \& Peñuelas, J., 1989. Water flow, trophic depletion, and benthic Macrofauna impoverishment in a submarine cave from the western Mediterranean. Marine Ecology, 10, 271-287.

Submitted 22 July 2003. Accepted 20 April 2004.
Appendix. List of species and authorities

\section{Div. CHLOROPHYTA}

Acetabularia acetabulum (Linnaeus) Silva

Acetabularia parvula Solms-Laubach

Anadyomene stellata (Wulfen) C. Agardh

Cladophora pellucida (Hudson) Kützing

Codium bursa J. Agardh

Flabellia petiolata (Turra) Nizamuddin

Halimeda tuna (Ellis \& Solander) Lamouroux

Halicystis parvula Schmitz

Palmophyllum crassum (Naccari) Rabenhorst

Pseudochlorodesmis furcellata (Zanardini) Børgesen

Valonia utricularis (Roth) C. Agardh

\section{Div. PHAEOPHYTA}

Colpomenia sinuosa (Mertens ex Roth) Derbès \& Solier in Castagne

Cystoseira balearica Sauvageau

Cystoseira compressa (Esper) Gerloff \& Nizamuddin

v. pustulata Ercegovic

Dictyopteris polypodioides (Stackhouse) Batters

Dictyota dichotoma (Hudson) Lamouroux

Dictyota dichotoma (Hudson) Lamouroux v. intricata

(C. Agardh) Greville

Dictyota fasciola (Roth) Howe

Halopteris filicina (Grateloup) Kützing

Halopteris scoparia (Linnaeus) Sauvageau

Lobophora variegata (Lamouroux) Womersley

Padina pavonica (Linnaeus) Thivy

Sphacelaria cirrosa (Roth) C. Agardh

Taonia atomaria (Woodward) J. Agardh

\section{Div. RHODOPHYTA}

Amphiroa beauvoisii Lamouroux

Amphiroa cryptarthrodia Zanardini

Amphiroa rigida Lamouroux

Asparagopsis armata Harvey

Boergeseniella fruticulosa (Wulfen) Sprengel

Bornetia secundiflora (J. Agardh) Thuret

Botryocladia boergesenii J. Feldmann

Botryocladia botryoides (Wulfen) J. Feldmann

Contarinia squamariae (Meneghini) Denizot

Corallina elongata Ellis \& Solander

Cryptomenia lomation (Bertoloni) J. Agardh

Falkenbergia rufolanosa (Harvey) Scmitz (life-history phase)

Gloiocladia furcata (C. Agardh) J. Agardh

Haliptilon virgatum Ellis \& Solander

Hydrolithon farinosum (Lamouroux) Howe

fania rubens (Linnaeus) Lamouroux

Laurencia obtusa (Hudson) Lamouroux

Lithophyllum cabiochae (Dufour) Furnari, Cormaci \& Alongi

Lithophyllum dentatum (Kützing) Foslie sensu Hamel \& Lemoine

Lithophyllum incrustans Philippi

Mesophyllum alternans (Foslie) Cabioch \& Mendoza

Neogonolithion brassica-florida (Harvey) Setchell \& Mason

Peyssonnelia rosa-marina Boudouresque \& Denizot

Peyssonnelia squamaria (Gmelin) Decaisne

Plocamium cartilagineum (Linnaeus) Dixon

Polystrata fosliei (Weber van Bosse) Denizot

Rhodophyllis divaricata (Stackhouse) Papenfuss 
Rhodymenia ardissonei J. Feldmann

Wurdermannia miniata (Sprengel) J. Feldmann \& Hamel

Phylum GRANULORETICULOSA

Miniacina miniacena (Pallas, 1766)

\section{Phylum PORIFERA}

Aaptos aaptos (Schmidt, 1864)

Acanthella acuta Schmidt, 1862

Agelas oroides (Schmidt, 1864)

Aplysina cavernicola (Vacelet, 1959)

Axinella damicornis (Esper, 1794)

Cacospongia mollior Schmidt, 1862

Chelanoplysilla noevus (Carter,1876)

Chondrosia reniformis Nardo, 1833

Clathrina clathrus (Schmidt, 1864)

Cliona celata Grant, 1826

Cliona schmidti (Ridley, 1881)

Cliona viridis (Schmidt, 1862)

Crambe crambe (Schmidt, 1862)

Crella mollior Topsent, 1925

Dendroxea lenis (Topsent, 1892)

Diplastrella bistellata (Schmidt, 1862)

Dysidea avara (Schmidt, 1862)

Erylus euastrum (Schmidt, 1870)

Hemimycale columella (Bowerbank, 1874)

Hexadella pruvotii Topsent, 1905

Hippospongia communis (Lamarck, 1813)

Ircinia fasciculata (Pallas, 1766)

Ircinia oros (Schmidt, 1864)

Ircinia variabilis (Schmidt, 1862)

Leucosolenia variabilis Haeckel, 1870

Myceliospongia araneosa Vacelet \& Perez, 1998

Oscarella tuberculata (Schmidt, 1868)

Petrosia ficiformis (Poiret, 1879)

Phorbas fictitius (Bowerbank, 1866)

Phorbas tenacior (Topsent, 1925)

Pione vastifica (Hanckock, 1849)

Pleraplysilla spinifera (Schulze, 1879)

Raspaciona aculeata (Johnston, 1842)

Reniera fulva Topsent, 1893

Reniera mucosa Griessinger, 1971

Reniera sarai (Pulitzer-Finali)

Spirastrella cunctatrix Schmidt, 1868

Spongia virgultosa (Schmidt, 1868)

Sycon elegans (Bowerbank, 1866)

Terpios fugax Duchassaing \& Michelotti, 1864

Topsentia garciae Bibiloni, Uriz \& Gili, 1989

\section{Phylum GNIDARIA}

Aglaophenia kirchenpaueri (Heller, 1868)

Alcyonium acaule Marion, 1878

Balanophyllia europaea (Risso, 1826)

Balanophyllia regia (Gosse, 1860)

Caryophyllia inornata (Duncan, 1878)

Cladocora caespitosa (Linnaeus, 1767)

Clavularia crassa (Milne-Edwards, 1848)
Corallium rubrum (Linnaeus, 1758)

Cornularia cornucopiae (Pallas, 1766)

Eunicella singularis (Esper, 1791)

Hoplangia durotrix Gosse, 1860

Leptopsammia pruvoti Lacaze-Duthiers, 1897

Maasella edwardsi (Lacaze-Duthiers, 1888)

Parerythropodium coralloides (Pallas, 1766)

Parazoanthus axinellae (Schmidt, 1862)

Polycyathus muellerae (Abel, 1959)

Sertularella ellisi (Milne-Edwards, 1836)

Phylum ANNELIDA

Myxicola aesthetica Koch in Renier, 1847

Salmacina dysteri (Huxley, 1855)

Serpula vermicularis Linnaeus, 1767

Phylum MOLLUSCA

Bittium reticulatum (Da Costa, 1778)

Lima hians (Gmelin, 1790)

Lithophaga lithophaga (Linnaeus, 1758)

Phylum EGTOPROCTA

Annectocyma indistincta (Canu \& Bassler, 1929)

Bugula calathus (Norman 1868)

Chlidonia pyriformis (Bertoloni, 1810)

Disporella hispida (Fleming, 1828)

Frondipora verrucosa (Lamouroux, 1821)

Idmidronea atlantica (Canu \& Bassler, 1928)

Lichenopora radiata (Audouin, 1826)

Margaretta cereoides (Ellis \& Solander, 1786)

Myriapora truncata (Pallas, 1766)

Parasmittina tropica (Waters, 1909)

Puellina gattyae (Landsborough, 1852)

Reptadeonella violacea Johnston, 1847

Schizomavella cuspidata (Hincks, 1880)

Schizomavella linearis (Hassall, 1841)

Smittina cervicornis (Pallas, 1766)

Smittoidea reticulata (MacGillivray, 1842)

Spiralaria gregaria (Heller, 1867)

Phylum BRACHIOPODA

Argyrotheca cordata (Risso, 1826)

Crania anomala (Müller, 1776)

Megerlia truncata (Linnaeus, 1767)

Phylum ECHINODERMATA

Paracentrotus lividus (Lamarck, 1816)

Phylum CHORDATA

Ascidia mentula Müller, 1776

Botrylloides leachi (De Savigny, 1816)

Clavelina lepadiformis (Müller, 1776)

Cystodytes dellechiajei (Della Valle, 1877)

Diplosoma spongiforme (Giard, 1872)

Lissoclinum perforatum (Giard, 1872)

Pyura dura (Heller, 1877) 